\title{
Sub- $\lambda / 2$ Displacement Sensor with Nanometric Precision based on Optical Feedback Interferometry used as a Non-Uniform Event-Based Sampling System
}

\author{
Olivier D. Bernal, Member, IEEE, Usman Zabit, Senior Member, IEEE, \\ Francis Jayat, and Thierry Bosch, Senior Member, IEEE, \\ https://doi.org/10.1109/JSEN.2020.2970599
}

\begin{abstract}
In this paper, a method based on the inherent event-based sampling capability of the laser optical feedback interferometry (OFI) is proposed to recover sub- $\lambda / 2$ displacement with a nanometric precision. The proposed method operates in open-loop configuration and relies on OFI's fringe detection, thereby improving its robustness and ease of use. The measured white noise power spectral density is less than $100 \mathrm{pm} / \sqrt{\mathrm{Hz}}$ with a corner noise frequency of approximately $80 \mathrm{~Hz}$ for a laser diode emitting wavelength $\lambda_{0}$ of $785 \mathrm{~nm}$ placed at $30 \mathrm{~cm}$ of the target. https://doi.org/10.1109/JSEN.2020.2970599
\end{abstract}

Index Terms-Optical feedback interferometry, self-mixing, displacement measurement, non-uniform sampling.

\section{INTRODUCTION}

O PTICAL feedback interferometry (OFI), also referred to as self-mixing (SM) effect in laser diodes (LD) [1], [2], [3] has been widely investigated for the last decades as it results in a self-aligned and cost effective sensing system. The resolution of a stationary OFI based displacement sensor depends on the employed signal processing techniques. Displacement measurement with a basic resolution of halfwavelength $\left(\lambda_{0} / 2\right)$ can be easily achieved with an OFI sensor under moderate optical feedback regime by fringe counting [1]. The basic resolution can be improved by locking the laser phase to half-wavelength [4] or by fringe duplication [5], [6] or by utilizing phase unwrapping techniques. Different phase unwrapping techniques (based on time-domain OFI signal processing) have been proposed in literature [7], [8], [9], [10], [11], [12], [13] providing accuracy from $\lambda_{0} / 8$ to $\lambda_{0} / 60$. For accuracy exceeding $\lambda_{0} / 40$, these methods [8], [9] require elaborate time-domain SM signal segmentations as well as estimations of key OFI parameters, such as optical feedback coupling parameter $C$. Except for the fringe-locking method [4], [14], [15], to the best of our knowledge, none of the previously mentioned methods exploiting the modulation of optical output power by OFI (also referred to as amplitude modulation (AM) channel) have achieved precision down to the nanometer yet.

O. D. Bernal, F. Jayat and T. Bosch are with the LAAS-CNRS, University of Toulouse, INP-ENSEEIHT, 31000 France e-mail: olivier.bernal@ toulouseinp.fr.

U. Zabit is with National University of Sciences and Technology, NUST, Islamabad, 44000, Pakistan.

DOI:10.1109/JSEN.2020.2970599
More recently, new methods exploiting the frequency modulation (FM) channel of OFI instead of the AM channel have shown that it is possible to further significantly improve the vibrometer performances by at least two decades [16]. However, to achieve these performances, it is required to convert FM-to-AM via narrow band optical frequency filter such as gas cell [17] or Mach Zehnder [18], which might hinder the simplicity of OFI systems.

Subsequently, using the AM SM signal alone, we do propose here a new open-loop approach that allows to recover sub- $\lambda_{0} / 2$ displacement with nanometric precision without requiring any closed-loop scheme for an LD of wavelength $\lambda_{0}$. This approach retains the inherent simplicity of OFI as the required hardware consists only in amplifying and acquiring the SM signal contrary to [19]. Further, as opposed to [4], in open-loop configuration, the distance $D_{0}$ from the LD to the target at rest neither directly affects the measurement precision nor the dynamic range. $D_{0}$ can affect the quality of the SM signals in terms of noise and $C$. However, only the change in the external cavity length corresponding to the target vibration will be embedded in the OFI signal. On the contrary, in closed-loop configuration, the loop compensates any target displacement by applying the required wavelength variation which is inversely proportional to $D_{0}$ [4], in order to maintain the interferometer phase constant. In addition, in this configuration, the achievable dynamic range is limited neither by the tunability range of the LD wavelength $\lambda_{0}$ nor by the finite value of the architecture's open-loop gain.

Here, in the moderate feedback regime $(1<C<4.6)$, we propose to perceive SM interferometers as an inherent nonuniform sampling system with its own embedded phase levelcrossing detector. Non-uniform sampling (NUS) approaches are often used in applications for which the retrieved information is sparse. Based on the non-uniform sampling theory, we show that it is possible to reconstruct the target displacement based on fringe detection only. Consequently, phase unwrapping techniques are not necessary to achieve nanometric precision. In addition, to recover sub- $\lambda / 2$ displacements for which a maximal of only one level crossing can be detected, we propose to add a phase dither $\Phi_{d}$, either obtained by vibrating the sensor itself or by modulating the LD driving current, to the SM phase so that both the number of crossed 


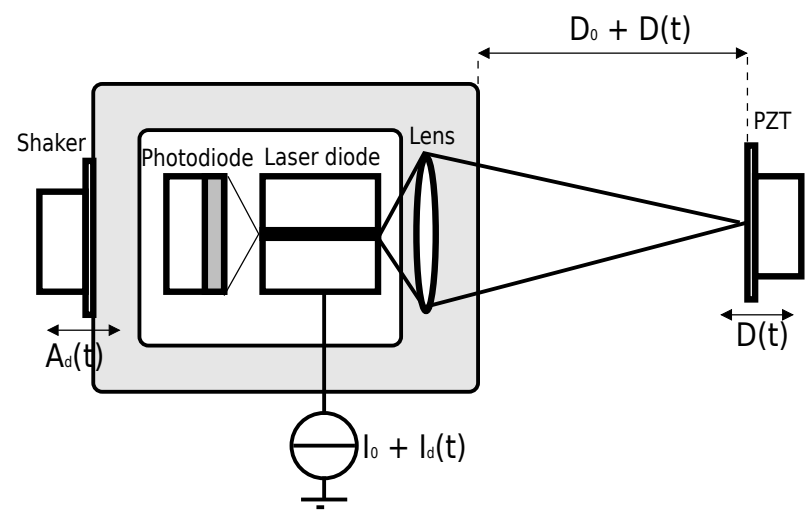

Fig. 1. Self-Mixing displacement sensor set-up with a piezoelectric transducer (PZT) used as a target. A dithering signal can be added either via the laser drive current $I_{0}+I_{d}(t)$ or via a shaker $A_{d}(t)$.

levels as well as the rate of level crossings can be increased. We will also show that using the NUS theory, estimating $C$ is not necessary when using dithering.

Note that dithering techniques were already employed in OFI to achieve high displacement resolution [20], [21] when combined to lock-in techniques. In [20], OFI is employed to design a simple and compact scattering-type scanning nearfield optical microscopy. However, the quantum cascade laser was operated at low temperature $(\approx 15 \mathrm{~K})$ in the very weak feedback regime $(C<0.1)$ and the dithering signal provided by a resonant vibrating tip was employed to allow amplitude and phase information retrieval on the medium optical response under test by applying lock-in techniques. In [21], the dithering signal is required for displacements both larger and smaller than $\lambda / 2$. It is obtained using an electrooptic modulator operating at $20 \mathrm{kHz}$ up to $0.2 \mathrm{MHz}$ while the $\mathrm{LD}$ is working in weak feedback regime. The instantaneous interference phase change related to the target displacement is retrieved by calculating the arc-tangent of the ratio between the zeroth-order and first-order harmonic amplitudes of the SM signal. These amplitudes are also obtained using lockin techniques with the dithering signal and its corresponding phase quadrature signal.

In the following section II, we present the non-uniform sampling theory applied on SM signals. We show how by applying a dithering signal, sub- $\lambda / 2$ displacements can also be recovered. Then, in section III, different experimental testbenches are described and results are analyzed to assess the system performances. Finally, effects of speckle on the achieved performances are discussed in section IV.

\section{Proposed Method}

In this section, a short background on OFI as well as on uniform and non-uniform sampling is first provided in order to introduce the proposed interpretation of OFI as an inherent non-uniform sampling system.

\section{A. OFI overview}

In OFI, a portion of the laser beam can be back-scattered from target placed at a distance $D_{0}$ from the laser (moving
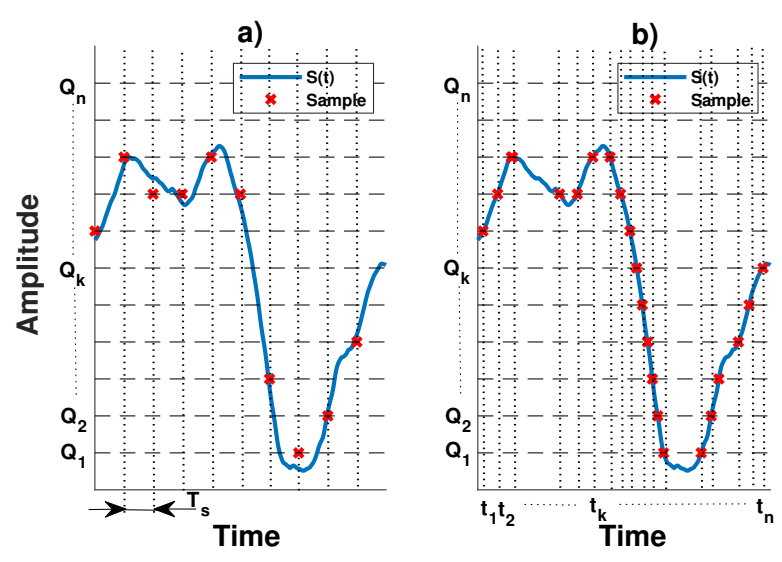

Fig. 2. Uniform sampling and quantization of $S(t)$ a) and non-uniform sampling of $S(t)$ in a level-crossing ADC b).The samples are shown as red crosses.

with displacement $D(t)$ ) and can thus re-enter the active laser cavity (Fig. 1). This causes a mixing of generated and phase-shifted back-scattered beams. This "self-mixing" causes fluctuation in the optical output power (OOP) of the laser, denoted as $P(t)$, given by [1]:

$$
P(t)=P_{0}\left(1+m \cos \left(\Phi_{F}(t)\right)\right)
$$

where $P_{0}$ is the emitted optical power under free-running conditions, $m$ is the modulation index and $\Phi_{F}(t)$ is the laser output phase in the presence of feedback. $\Phi_{F}(t)$ is related to the laser output phase without feedback $\Phi_{0}(t)=4 \pi D(t) / \lambda_{0}$ by:

$$
\Phi_{0}(t)=\Phi_{F}(t)+C \sin \left(\Phi_{F}(t)+\arctan \alpha\right)
$$

where $\alpha$ is referred to as the linewidth enhancement factor [1], [2]. Depending on $C$, the laser can operate into different regimes. SM sensing is generally performed under weak feedback regime $(C<1)$, moderate feedback regime $(1<C<4.6)$, or strong feeback regime $(C>4.6)$. However, moderate feedback regime $(1<C<4.6)$ is usually preferred as the apparently simple saw-tooth shaped SM fringes belonging to such a regime [22] intrinsically provide motion direction indication and require simplified SM fringe detection processing [23].

\section{B. Uniform and non-uniform sampling principles}

A discrete-time representation of a band-limited signal $S(t)$ is usually obtained using an analog-to-digital converter (ADC) which uniformly samples $S(t)$ at a frequency $f_{s}=1 / T_{s}$ and quantizes it to the nearest quantization level $\left(Q_{1} \ldots Q_{n}\right)$ [24], [25]. ADCs thus represent the signal in time-amplitude ordered pairs $\left[k T_{s}, Q_{k}\right]$ with $Q_{k}$ the nearest quantization level at $k T_{s}$ (see Fig. 2 a)). As shown in Fig. 2 a), the quantization process induces an estimation error of the correct amplitude of $S(t)$. To reduce the quantization error and improve the dynamic range without increasing the number of quantization levels, oversampling techniques can be used in order to distribute the quantization error power over a larger bandwidth than the signal of interest [24]. While the quantization process of the previously described ADC's scheme is clock driven, ADCs 


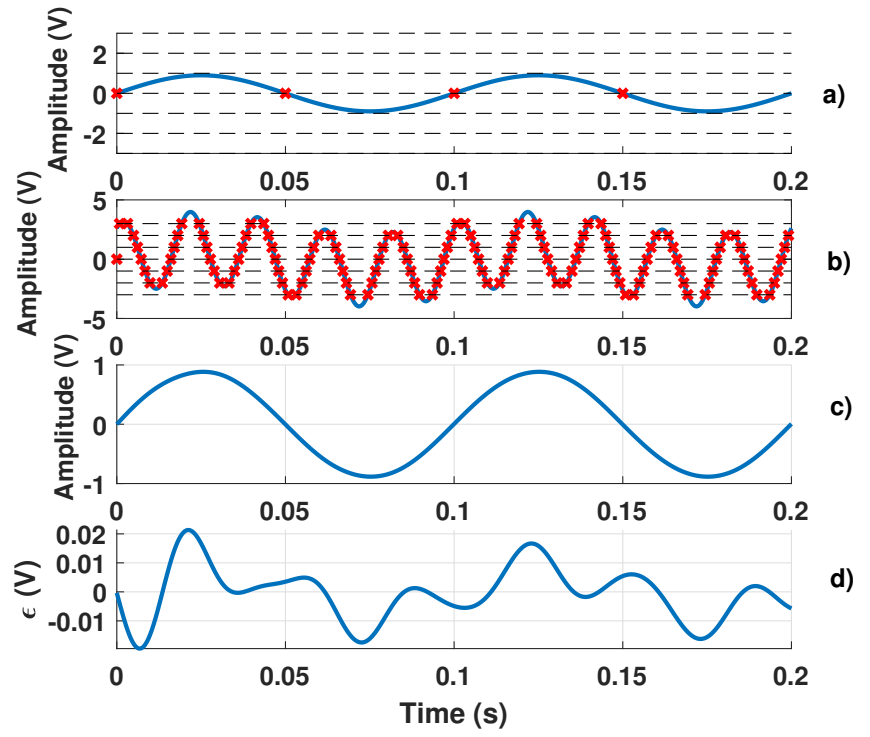

Fig. 3. Simulated level-crossing ADC: a) a $0.9 \mathrm{~V}$ sinusoidal input $S(t)$ at $10 \mathrm{~Hz}$ crosses only the $0 \mathrm{~V}$-threshold level, b) after adding a $3 \mathrm{~V} 50 \mathrm{~Hz}$ dithering signal to $S(t)$ many more samples are generated, c) reconstructed input signal using spline interpolation after having subtracted the dithering signal and d) the remaining reconstruction error.

can be designed to be event driven instead. These ADCs are often referred as level crossing ADCs which record the time instants $t_{k}$ at which $S(t)$ crosses any of its quantization levels $Q_{k}$ resulting in time-level pairs $\left[t_{k}, Q_{k}\right]$ (see Fig. 2 b)) [24], [25]. The time instants $t_{k}$ of the level-crossings are then quantized into $Q\left(t_{k}\right)$ using a clock that must provide both a high resolution and precision. As a result, if the average sampling rate of the input exceeds twice the input signal bandwidth, it is possible to retrieve the original band-limited signal $S(t)$ from the non-uniformly spaced in time samples $\left[Q\left(t_{k}\right), Q_{k}\right][26]$.

In addition, in order to both improve the dynamic range of the level-crossing ADCs without increasing the number of level-crossings and fulfill the requirements on the average sampling rate of level crossings, adding a dithering signal to the input signal can be employed to ensure that low-amplitude (lower than the quantization levels) or slowly varying signals are sampled and converted accurately [27], [28]. This dithering principle can be illustrated by Fig. 3 where a $10 \mathrm{~Hz} 0.9 \mathrm{~V}$ sinusoidal input $S(t)$ is applied at the input of a level-crossing level ADC. Fig. 3 a) shows that it crosses only one of the threshold levels uniformly-spaced by $1 \mathrm{~V}$, which results in a poor analog-to-digital conversion. However, if a $50 \mathrm{~Hz}$ $3 \mathrm{~V}$ sinusoidal dithering signal is added to $S(t)$, a lot of samples are generated (Fig. 3 b)). Consequently, $S(t)$ can be reconstructed using spline interpolation for instance after having removed the dithering signal with a small remaining reconstruction error. The level-crossing ADC principle can be summarized by the block diagram shown in Fig. 4.

\section{OFI as a non-uniform sampling system}

Based on the previous description of level-crossing ADCs, in the moderate feedback regime, based on (1) and (2), we

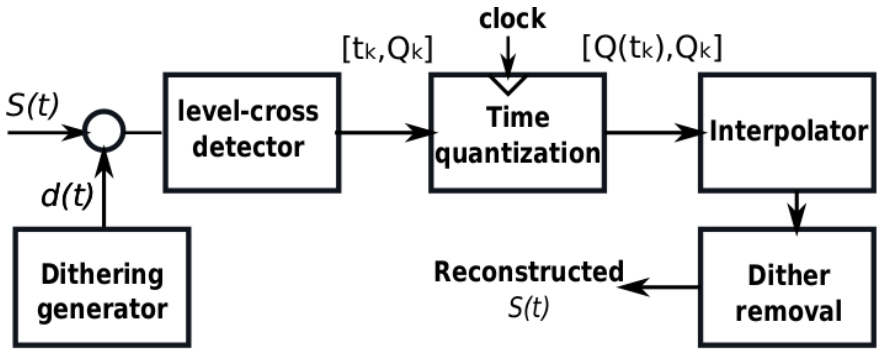

Fig. 4. Block diagram of a typical level-crossing ADC

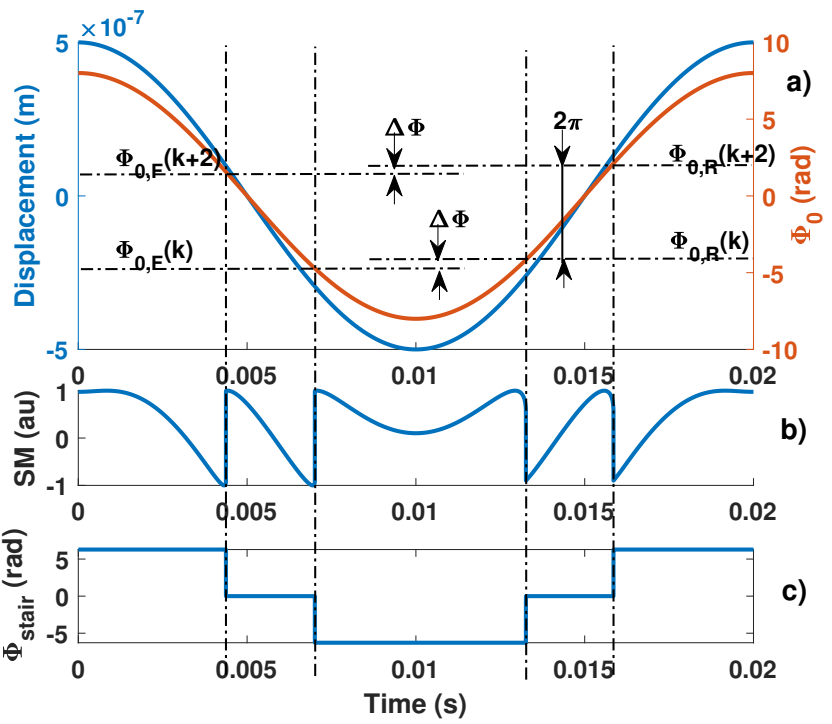

Fig. 5. Simulated typical Self-Mixing signal (b) obtained for (a) a $0.5 \mu \mathrm{m}$ sinusoidal displacement, a laser wavelength $\lambda_{0}=785 \mathrm{~nm}$ and $C=1.5$ (in blue line) with its corresponding phase $\Phi_{0}$ (in red) and (c) staircase phase $\Phi_{\text {stair }}$

propose to perceive SM interferometers as an inherent nonuniform sampling system with its own embedded phase levelcrossing detector. The information on $D(t)$ is completely enclosed within the phase $\Phi_{F}$ (2). By monitoring the OOP discontinuities, a phase domain level crossing every $2 \pi$ can thus be obtained (Fig.5). Further, contrary to classical levelcrossing analog-to-digital converters (ADCs), the number of phase quantization levels (PQL) $\Phi_{0}(k)$ is ideally infinite due to the folding or wrapping occurring every $2 \pi$ introduced by the cosine in the OOP (see (1) and [29]). However, as shown in Fig.5, these phase quantization levels $\Phi_{0}(k)$ are slightly different (by an amount denoted $\Delta \Phi$ ) for an increasing and decreasing $\Phi_{0}$ phase. These PQLs can thus be referred to as $\Phi_{0_{R}}$ and $\Phi_{0_{F}}$ when $\Phi_{0}$ is increasing or decreasing respectively. They are completely defined by (2) with $\Phi_{F}$ as given in [29] whenever $\Phi_{F}$ has infinite slopes:

$$
\begin{aligned}
& \Phi_{F, R}=k \pi-\arctan (\alpha)+\arccos \left(\frac{-1}{C}\right) \\
& \Phi_{F, F}=(k+2) \pi-\arctan (\alpha)-\arccos \left(\frac{-1}{C}\right)
\end{aligned}
$$

where $k$ is an even integer. It will be later shown that this $\Delta \Phi$ does not cause any issue for the proposed approach and can 
be expressed as a function of $C$ :

$$
\Delta \Phi=2\left(\arccos \left(\frac{-1}{C}\right)+\sqrt{C^{2}-1}-\pi\right)
$$

The SM phase level-crossing detector outputs time-phase pairs $\left[t_{n}, \Phi_{n}\right]$. For each pair, $t_{n}$ corresponds to the time instant when $\Phi_{0}(t)$ crosses one PQL which can be either $\Phi_{0_{R}}(k)$ or $\Phi_{0_{F}}(k)$. Since these pairs $\left[t_{n}, \Phi_{n}\right]$ must be recorded, these time instants are quantized $Q\left(t_{n}\right)$ with a time resolution of $1 / f_{s}$ (where $f_{s}$ is the sampling frequency of the data acquisition system) to generate non-uniform samples (NUS) $\left[Q\left(t_{n}\right), \Phi\left(t_{n}\right)\right]$. As shown in [26], the continuous time input signal can be reconstructed from these samples if the quantization sampling rate of the input signal exceeds twice the input signal bandwidth. In addition, to be further processed, these $\left[Q\left(t_{n}\right), \Phi\left(t_{n}\right)\right]$ sets are usually fed to an interpolator to generate a uniformly sampled rate output signal. However, for sub- $\lambda_{0} / 2$ displacements, none or only one level crossing (depending on the initial phase value of $\Phi_{F}$ ) can be detected thereby leading to a poor displacement reconstruction.

\section{Dithering and sub- $\lambda_{0} / 2$ displacement recovery}

Here, in a manner similar to approaches used in NUS ADCs, a phase dither $\Phi_{d}$ can be added to the phase $\Phi_{0}$ so that both the number of crossed levels as well as the rate of level crossings can be increased. The resulting equivalent displacement $D_{\Sigma}(t)$ is obtained by summing the target displacement $D(t)$ to the equivalent dither displacement $D_{d}(t) . \Phi_{d}$ can be implemented either by directly vibrating the laser itself with $D_{d}(t)$ or indirectly by modulating the laser driving current, thereby modulating $\lambda_{0}$. Here, a cosine vibration dithering has been chosen with an amplitude $A_{d}$ and a frequency $f_{d}$. To achieve an accurate displacement reconstruction, the average nonuniform sampling rate should be at least twice the $D_{d}(t)$ bandwidth according to the Nyquist criterion. Consequently, $f_{d}$ can be chosen to be greater than the bandwidth of interest and $A_{d}$ should then be chosen greater than $\lambda_{0} / 2$ to induce at least two level crossings, thereby guaranteeing the Nyquist criterion. Note that this criterion can also be satisfied with lower $f_{d}$ values associated with correspondingly higher $A_{d}$ values. However, it makes it more difficult to retrieve the target displacement as the dithering signal would then be within the bandwidth of interest. As a result, here $f_{d}$ is chosen greater than the displacement bandwidth of interest so that it is not required to precisely know $A_{d}$ (it should only be $>\lambda_{0} / 2$ ).

If the Nyquist criterion is satisfied then the band-limited displacement $D(t)$ can be reconstructed from the non-uniform samples (NUSs). As previously mentioned, to achieve this reconstruction, interpolators are required to convert these NUSs into uniform samples. Different interpolators can be employed to fulfill this task such as: splines, polynomials [26], sinc-based polynomials [25]... In addition, if the SM signals are acquired using an $\mathrm{ADC}$, then phase unwrapping algorithms correspond to the perfect interpolator. Here, the Consecutive Samples based Unwrapping (CSU) approach that consists in adding the normalized SM signal to the staircase approximation $\Phi_{\text {stair }}$ of $\Phi_{0}$ based on $\left[Q\left(t_{n}\right), \Phi\left(t_{n}\right)\right]$, is used

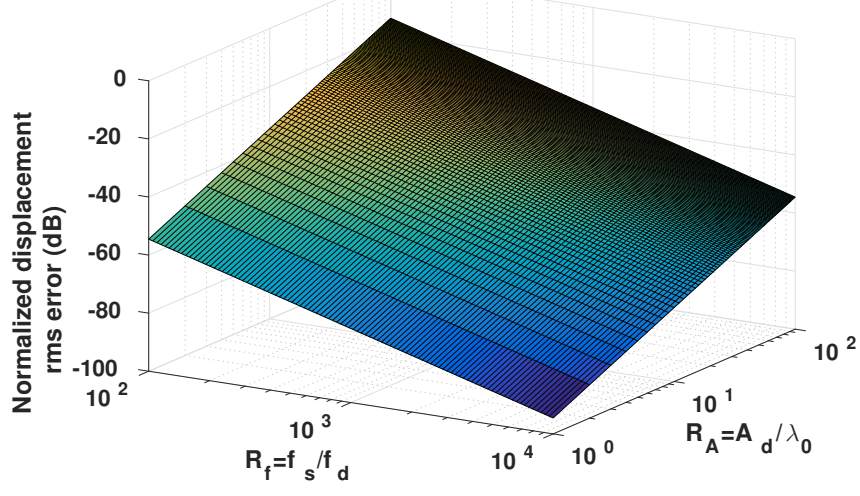

Fig. 6. Normalized Displacement RMS error to $\lambda_{0}$ induced by the time instants quantization through $R_{f}=f_{s} / f_{d}$ and the dither signal amplitude through $R_{A}=A_{d} / \lambda_{0}$

as interpolator since it is an attractive alternative with respect to reduced computational requirements [12].

The achieved reconstruction performance depends directly on the number of effective quantization levels (NEQLs) $N$ [24], and on the accuracy of both the quantization levels $\delta \Phi$ and of $f_{s}$. Based on [24], [28], the combined root mean square (rms) displacement quantization error $\epsilon_{r m s}$ per sample due to $f_{s}$ and $\delta \Phi$ can be expressed as:

$$
\epsilon_{r m s}=\frac{1}{2 \pi} \frac{\lambda_{0}}{2} \sqrt{\frac{\delta \Phi^{2}}{3}+\frac{16 \pi}{9}\left(\frac{A_{d} f_{d}}{\lambda_{0} f_{s}}\right)^{2}}
$$

(6) and Fig. 6 clearly show that a high ratio $R_{f}$ of the sampling frequency $f_{s}$ to the dither signal frequency $f_{d}$ is required to achieve low displacement errors while the ratio $R_{A}=A_{d} / \lambda_{0}$ can be kept small. Even if a higher value of $A_{d}$ results in a higher number of level crossings, a higher value of $A_{d}$ necessitates higher $f_{s}$ value to keep decreasing $\epsilon_{r m s}$ [24], [28]. Further, for high $A_{d}$, the SM signal might suffer from speckle effects [30]. Here $A_{d}$ is thus chosen to be equal to a few $\lambda_{0}$ only since even with a low number of quantization levels a high resolution can still be achieved [24]. As a result, the accuracy of the system is mainly dependent on $\delta \Phi$ for which different noise sources can be identified [4]: the LD linewidth [31], the mechanical noise of the experimental setup, the LD driving current noise and the temperature noise that can both affect $\lambda_{0}$. Fig. 7 shows the effect of NEQLs on the displacement reconstruction error using the CSU. As expected, this error increases with $C$ [12] and decreases with increasing dithering amplitudes [24].

\section{E. Impact of the Optical Feedback Factor $C$ on the recon- structed displacement}

As previously mentioned, it is important to also note that as shown in Fig. 5, SM interferometers cannot be considered as ideal NUS systems since their PQL $\Phi_{0}(k)$ are slightly different (by an amount denoted $\Delta \Phi$ ) for an increasing and decreasing $\Phi_{0}$ phase (see (8)). It is thus clear that even if $\Phi_{\text {stair }}$ is usually used as a rough estimation of $\Phi_{0}, \Phi_{\text {stair }}$ 

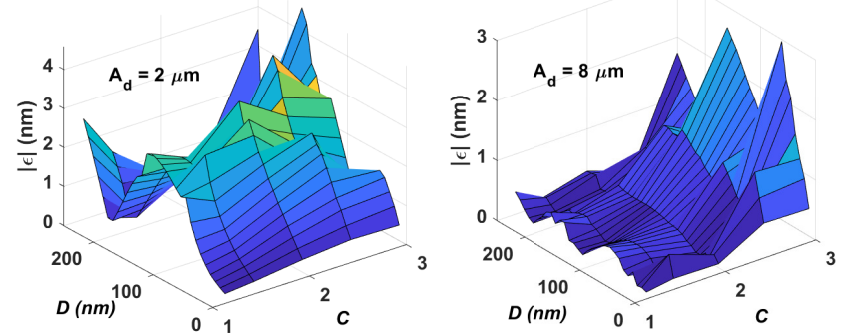

Fig. 7. Absolute displacement error retrieved with the CSU interpolating method vs different $C$ values and the target displacement amplitudes $D$ for different dithering amplitude $A_{d}$

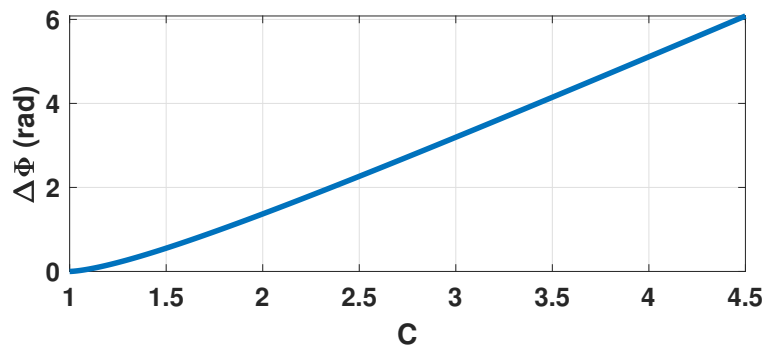

Fig. 8. Simulated difference between increasing and decreasing phase value $(\Delta \Phi)$ as a function of $C$ as per (2)

does not take into account $\Delta \Phi$ and hence inherently contains some errors.

Consequently, it strongly suggests that not taking into account $\Delta \Phi$ might result in reduced performances if $C$ is not considered. To estimate this error, it is interesting to look at this issue from a different perspective. Instead of considering the PQLs $\Phi_{0_{R}}$ and $\Phi_{0_{F}}$ to be different, they can be supposed to be equal if a virtual square displacement of amplitude $\lambda_{0} \Delta \Phi / 8 \pi$ is added on top of $D_{d}$ at $f_{d}$. Fig 9 shows the simulated results obtained for a SM signal corresponding to a $4 \mu \mathrm{m}$ sinusoidal displacement for $C=2$ with its corresponding erroneous $\Phi_{\text {stair }}$, its ideal $\Phi_{\text {stair }}$ and the reconstructed displacement errors using Spline interpolation in both cases. The maximum error $\epsilon_{\max } \approx 42 \mathrm{~nm}$ is thus in accordance with the predicted error $\lambda_{0} \Delta \Phi / 8 \pi$. Fig. 10 shows the influence of the $C$ value on the displacement error. Using a spline interpolator and the non-uniform sampling theory, the RMS error of the reconstructed displacement $\epsilon_{r m s}$ is $2.2 \mathrm{~nm}$ and $40.7 \mathrm{~nm}$ with (by adding or subtracting $\Delta \Phi / 2$ to $\Phi_{0_{R}}$ or $\Phi_{0_{F}}$ respectively if $C$ is known [7], [9], [8], [32]) and without $C$ taken into account respectively.

In presence of a dithering signal, the change of direction occurs at the dithering frequency $f_{d}$, which is outside of the signal bandwidth of interest. Consequently, $\Delta \Phi$ has a direct impact on the retrieved amplitude of the dithering signal $D_{d}$ but none on the desired displacement $D(t)$. Fig. 11 shows the reconstructed displacement spectrum with and without a $2 \mu \mathrm{m} 1 \mathrm{kHz}$ dithering signal for a $4 \mu \mathrm{m} 90 \mathrm{~Hz}$ sinusoidal displacement using Spline interpolation. It clearly shows that unwanted harmonics generated by $\Delta \Phi$ are efficiently removed
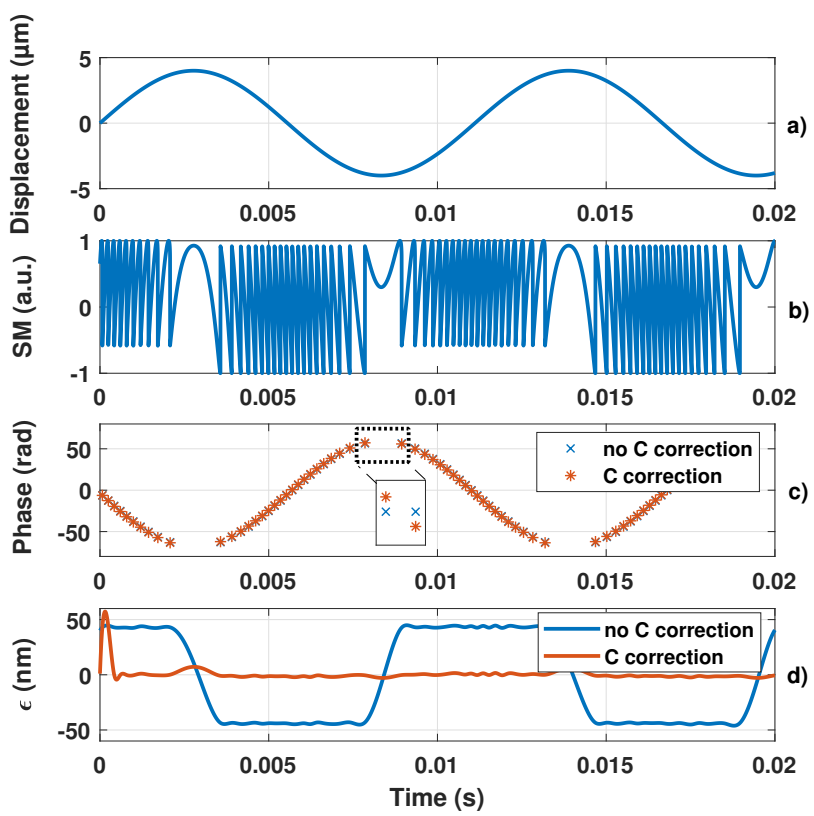

Fig. 9. Simulated SM signal (b) obtained for a $4 \mu m$ displacement at $90 \mathrm{~Hz}$ with $C=2$ (a) with a laser diode operating with a $\lambda=785 \mathrm{~nm}$. Reconstructed $\Phi_{\text {stair }}(\mathrm{c})$ by discontinuity detection with (red) and without (blue) $C$ value taken into account. Obtained reconstructed displacement error $\epsilon$ induced with (red) and without (blue) $C$ taken into account while using the non-uniform sampling approach with a spline interpolator.

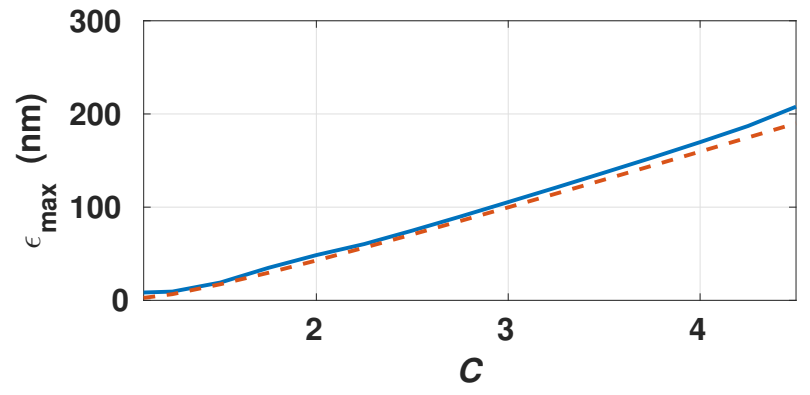

Fig. 10. Simulated displacement error $\epsilon$ (blue line) and estimated error $\lambda_{0} \Delta \Phi / 8 \pi$ (red dashed line) induced when $C$ is not taken into account in $\Phi_{\text {stair }}$ obtained for a $4 \mu \mathrm{m} 90 \mathrm{~Hz}$ displacement and spline interpolation.

by the dithering and appeared at frequency higher than $f_{d}$. This is also clearly shown in Fig.12: while Fig.12 d) illustrates that the $C$ induced error on the reconstructed signal (blue curve) is similar to the one obtained previously without dithering signal (Fig. 9), Fig.12 e) demonstrates that by removing all the signal frequencies $\geq f_{d}$, the remaining error is similar to the one obtained when $C$ is taken into account. In addition, the RMS error is $2.8 \mathrm{~nm}$ and $18 \mathrm{~nm}$ with and without dithering signal respectively over a $1 \mathrm{kHz}$ bandwidth, which is similar to the $2.2 \mathrm{~nm}$ RMS error obtained when $C$ is taken into account.

\section{RESULTS}

A SM test bench (see Fig.13) was developed to assess the performances of the proposed approach through two main test procedures. The aim of the first one is to verify the principle of dithering. In this case, the target generates both 


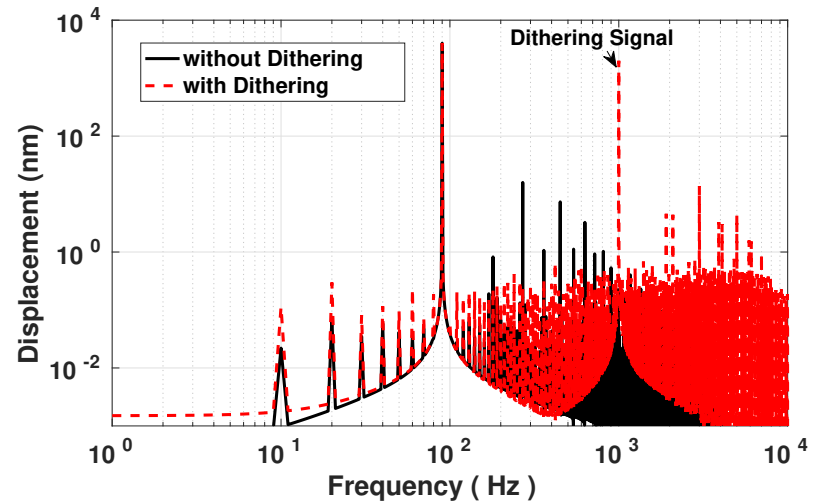

Fig. 11. Simulated reconstructed displacement frequency spectrum with (red dashed line) and without (black line) a dithering signal of $2 \mu m$ at $f_{d}=1 \mathrm{kHz}$ for $C=2$, a target displacement of a $4 \mu m$ and $90 \mathrm{~Hz}$ displacement and spline interpolation.

the dithering displacement and the sub- $\lambda_{0} / 2$ displacement while the laser diode remains stationary. In the second case, as a proof of concept, the target only generates the sub$\lambda_{0} / 2$ displacement to be recovered while the laser diode is vibrated at one of the mechanical resonance frequencies of the system by a shaker to generate the dithering displacement. The $\mathrm{LD}$, driven by a constant injection current of $30 \mathrm{~mA}$, is a Hitachi HL7851G emitting at $\lambda_{0}=785 \mathrm{~nm}$. The system benefits from the autofocus based on the liquid lens ARTIC 39N0 from Varioptic [32]. A piezoelectric transducer (PZT) from Physik Instrumente (P753.2CD) is used as a target positioned at $30 \mathrm{~cm}$ from the LD. It is equipped with an internal capacitive feedback position sensor with a $0.2 \mathrm{~nm}$ resolution and $2 \mathrm{~nm}$ repeatability. The data is acquired by a NI USB 6251 data acquisition system operating at $10^{6} \mathrm{sample} / \mathrm{s}$ with a 16 bit resolution.

Prior to any measurements, $\epsilon_{r m s}$ can be estimated through the system phase noise measurement based on [31] and (6). As a result, $\delta \Phi$ is estimated to be approximately $0.136 \mathrm{rad}$ corresponding to $\epsilon_{r m s} \approx 4.9 \mathrm{~nm}$.

Here, the data is firstly processed using the CSU method in order to reconstruct a first estimate of $D_{\Sigma}$. Then, an FFT analysis is performed to filter out all the signals out of the bandwidth of interest $\left(f \geq f_{d}\right)$. Figure 14 shows the measured amplitude of the displacement extracted from the SM signal acquired during $1 \mathrm{~s}$ in order to retrieve the sub- $\lambda_{0} / 2$ displacement of a target vibrating at $20 \mathrm{~Hz}$ while the dithering vibration is set at $A_{d}=2.5 \mu \mathrm{m}$ and $f_{d}=90 \mathrm{~Hz}$. As a consequence, $R_{f}$ is approximately $11 \mathrm{k}$ and $R_{A}$ is 1.6 corresponding to 13 NEQLs approximately. For each displacement amplitude, a set of 10 measurements is performed. Note that spline interpolation can also be used instead of CSU. However, the obtained performances are slightly better for CSU. Figure 15 shows a typical spectrum of the reconstructed displacement signal together with the dithering signal. The proposed method's performances for 3 different dithering amplitudes and $D$ $\in[2.5 \mathrm{~nm} ; 500 \mathrm{~nm}]$ are summarized in Table I in terms of the absolute mean error $|\epsilon|$, and standard deviation $\sigma$. It is interesting to note that the best result is not obtained for the
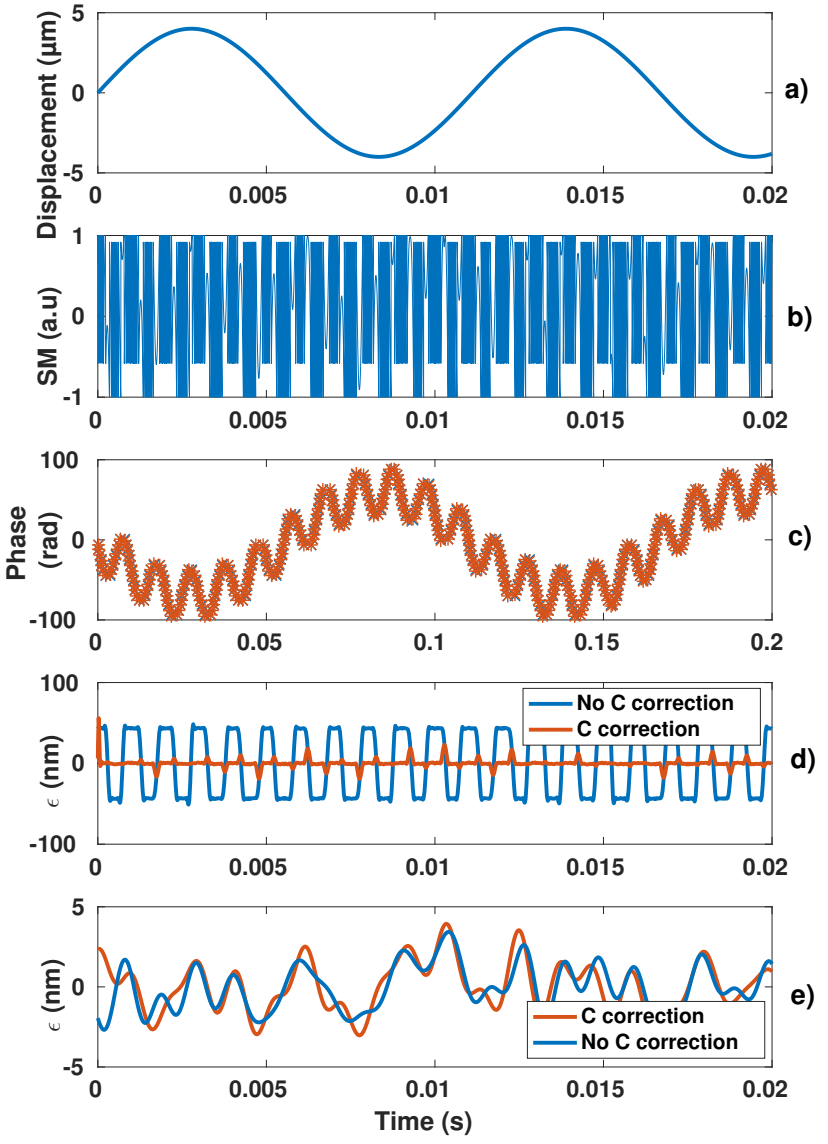

Fig. 12. Simulated SM signal (b) obtained for a $4 \mu m$ displacement at $90 \mathrm{~Hz}$ with $C=2$ (a) with a dithering signal of $2 \mu \mathrm{m}$ at $f_{d}=1 \mathrm{kHz}$ for a laser diode operating with a $\lambda=785 \mathrm{~nm}$. Reconstructed $\Phi_{\text {stair }}$ (c) by discontinuity detection with (red) and without (blue) $C$ value taken into account. Finally d), obtained reconstructed displacement error $\epsilon$ induced with (red) and without (blue) $C$ taken into account while using the non-uniform sampling approach with a spline interpolator and e) obtained from d) curves by removing all frequencies $f>f_{d}$.

TABLE I

Measured Displacement Reconstruction Performances vs Dithering Amplitude $A_{d}$ or NEQLs using the CSU interpolator with $1 \mathrm{~Hz}$ bandwidth FFT analysis

\begin{tabular}{cccc}
\hline$A_{d}(\mu m)$ & NEQLs & Mean $|\epsilon|(n m)$ & Mean $\sigma(n m)$ \\
\hline $2.5^{*}$ & 13 & 1.9 & 0.85 \\
$4.5^{*}$ & 22 & 2.2 & 1.4 \\
$1.2^{* *}$ & 6 & 1.7 & 0.96 \\
\hline
\end{tabular}

* PZT vibrating at $90 \mathrm{~Hz}$

** Shaker vibrating at $50 \mathrm{~Hz}$

highest NEQLs of 22 but for 13. This might be explained by the presence of a higher speckle level for the NEQLs of 22 that can introduce $C$ variations, thereby inducing some more phase errors.

Note that the proposed approach can be also applied to displacements larger than $\lambda_{0} / 2$ (Fig. 16) as even more PQLs would then be generated. The measured white noise power spectral density is less than $100 \mathrm{pm} / \sqrt{\mathrm{Hz}}$ with a corner 


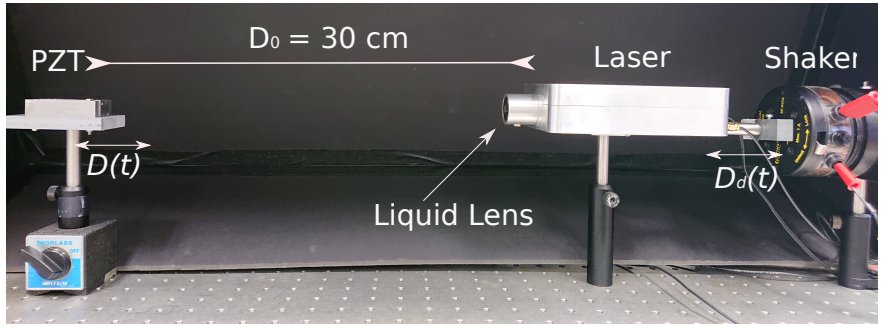

Fig. 13. The sub- $\lambda / 2$ SM displacement sensor test bench. Piezoelectric transducer (PZT) containing internal capacitive feedback sensor with $2 \mathrm{~nm}$ resolution is used to generate target displacement $D(t)$. Mechanical shaker provides the dithering vibration $D_{d}(t)$ to the SM sensor.
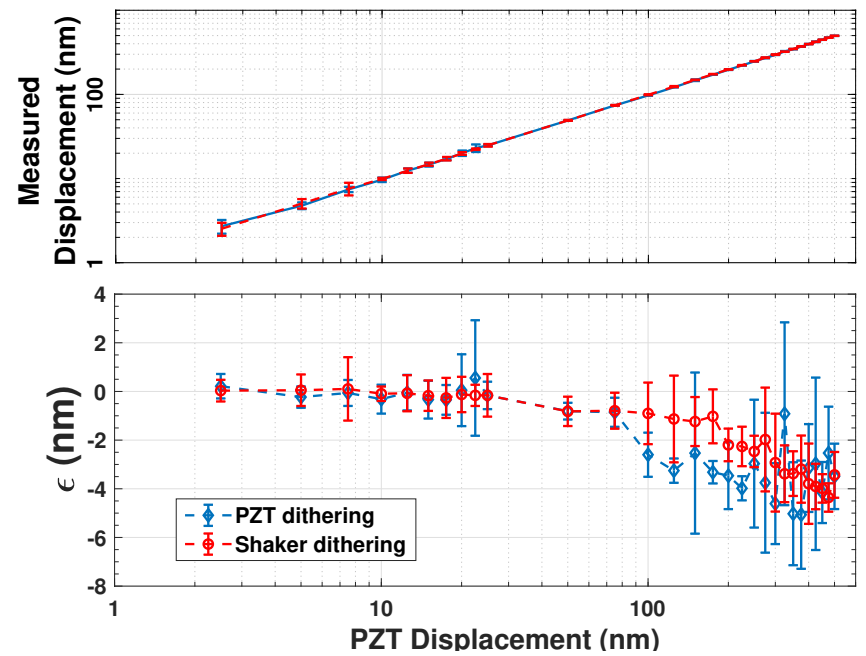

Fig. 14. Measured sub- $\lambda / 2$ displacement amplitude with HL7851 LD having $\lambda_{0}=785 \mathrm{~nm}$ placed at $30 \mathrm{~cm}$ from the PZT using the CSU interpolator via FFT analysis : (1) in blue line, PZT is used both as the target vibrating at $20 \mathrm{~Hz}$ and as the dithering reference vibration at $90 \mathrm{~Hz}$ with $A_{d}=4.5 \mu \mathrm{m}$ and (2) in red dashed line, the shaker generates the dithering vibration at $50 \mathrm{~Hz}$ with $A_{d}=1.2 \mu \mathrm{m}$.

frequency $f_{c}$ related to the $1 / \mathrm{f}$ noise estimated to be approximately $80 \mathrm{~Hz}$ (Fig.16). Finally, the proposed approach can also recover other kind of vibrations. For instance, Fig. 17 shows the reconstructed displacement of a damped harmonic vibration at $55 \mathrm{~Hz}$ compared to the PZT.

\section{Discussion And Perspectives}

For large displacements, $C$ might suffer slow drifts due to speckle. Subsequently, the PQL will also drift accordingly. Contrary to the precedent analysis on the impact of $C$ on the proposed method performances, this will then result in reconstruction error since the phase difference between two consecutive PQL of $\Phi_{0_{R}}$ and $\Phi_{0_{F}}$ are no more equal to $2 \pi$. To correctly recover the displacement, knowing $C$ real-time value is thus necessary. In another study, it will be shown that using our NUS method such estimation can be obtained contrary to all existing methods that only provide an average value of $C$.

However, note that except for the fringe locking method [4], all the other SM unwrapping based methods will suffer from

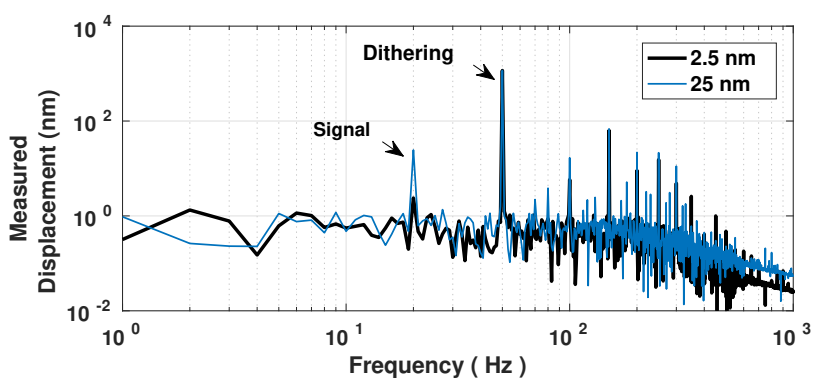

Fig. 15. FFT of the reconstructed displacement obtained with the spline interpolation for a $20 \mathrm{~Hz}$ target vibration with an $2.5 \mathrm{~nm}$ (black curve) and $25 \mathrm{~nm}$ (blue curve) amplitude and a $1.2 \mu \mathrm{m}$ dithering amplitude obtained by the shaker at $50 \mathrm{~Hz}$.

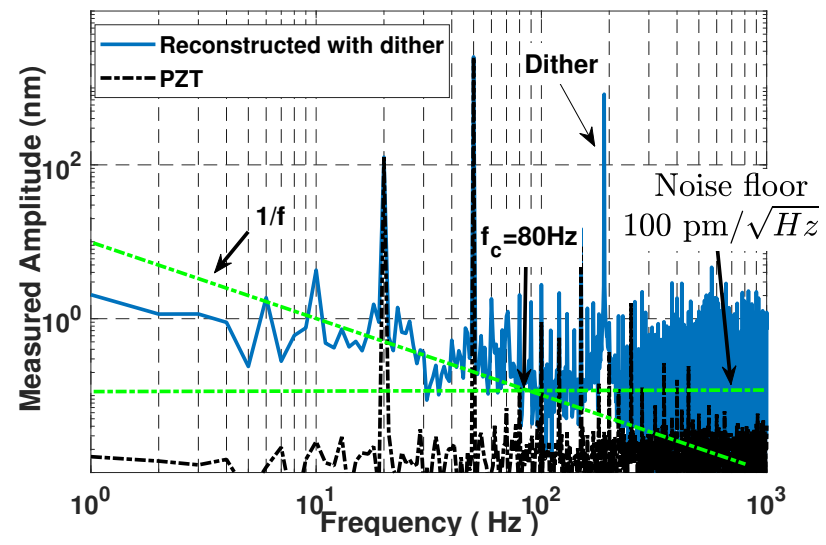

Fig. 16. Measured FFT of the reconstructed displacement $D(t)$ using the CSU interpolation for a $125 \mathrm{~nm}$ and $2.5 \mu \mathrm{m}$ amplitude target vibration at $20 \mathrm{~Hz}$ and $55 \mathrm{~Hz}$ respectively with a $0.8 \mu$ m dithering amplitude obtained by the shaker at $190 \mathrm{~Hz}$.

the same issues. Nonetheless, due to the inherent simplicity of the proposed approach and its ability to deal with sub- $\lambda_{0} / 2$ displacement (for which speckle is usually negligible), the proposed algorithm is still of great interest. In addition, Fig. 18 shows the evolution of the total rms error $\epsilon_{r m s}$ as a function of the $C$ modulation due to speckle $\left(C=C_{0}+C_{m} \sin (2 \pi f t)\right.$ where $C_{0}=2$ and $f$ is equal to the displacement frequency) and of the displacement amplitude $D$. Based on (6), without any $C$ modulation, the estimated $\epsilon_{r m s}$ is approximately $3.3 \mathrm{~nm}$. The results obtained in the presence of speckle show that the achievable performances are comparable to this figure for $C_{m}<0.3$.

As previously mentioned in section II, if $C$ is not taken into account, it generates virtual square displacement, the amplitude of which is related to (2). It can thus be possible to both detect and estimate it via the reconstructed displacement spectrum. In addition, the high dithering frequency compared to the slow varying $C$ value might give the opportunity to observe the evolution of $C$ with time, which might allow fine $C$ correction and real-time $C$ adjustment via adaptive optics [32]. 

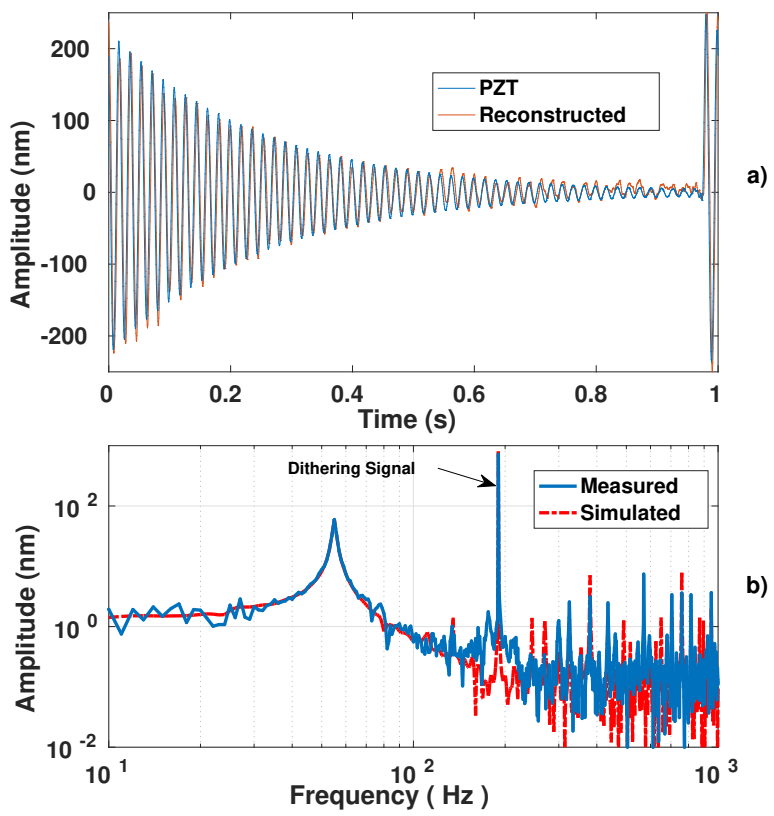

Fig. 17. Reconstructed damped harmonic displacement (red) for $A_{d}=0.8 \mu \mathrm{m}$ and $f_{d}=190 \mathrm{~Hz}$ compared to PZT displacement (blue) (a) and (b) FFT of the reconstructed measured (blue) and simulated (red) displacement both using the CSU.

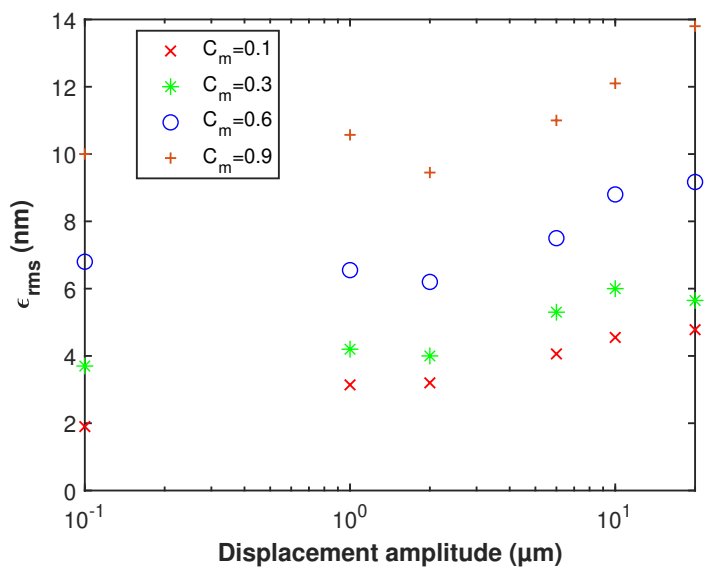

Fig. 18. Simulated total RMS error $\epsilon_{r m s}$ over the full bandwidth for $A_{d}=2 \mu \mathrm{m}$ at $200 \mathrm{~Hz}$ for different PZT displacement amplitudes at $\mathrm{f}=45 \mathrm{~Hz}$ and varying $C$ value described here as $C=C_{0}+C_{m} \sin (2 \pi f t)$.

\section{CONCLUSiON}

The proposed sensor can measure sub- $\lambda_{0} / 2$ displacement and achieve a high precision with a noise power spectral density $\leq 100 \mathrm{pm} / \sqrt{\mathrm{Hz}}$ while based on both a relatively simple set-up and processing method compared to [19]. We propose to interpret OFI as a non-uniform event based sampling system where the quantization levels are clearly defined by the signal discontinuities in the moderate optical feedback regime. It was shown that the CSU ([12]) can be used as an efficient interpolator. However, to design an even more efficient system, it might be interesting to choose another kind of mathematical interpolators since no analog-to-digital conversion would then be required. With the non-uniform sampling approach, the instants when quantization levels are crossed contain all the information. It is thus possible to envision the development of a system based only on fringe detection using an analog front-end similar to [33] and then process the acquired data. As a result, the amount of acquired data to be processed can be much less than that of a classical approach based on an ADC front-end, resulting in simpler and cheaper embedded monitoring systems. Based on this non-uniform event based proposed interpretation, a dithering signal was added here to successfully recover sub- $\lambda_{0} / 2$ displacements as well as higher amplitude ones. Up to now, the system's main limitation is the bandwidth (here $190 \mathrm{~Hz}$ corresponds to the maximal mechanical resonant frequency of the shaker-laser system) directly related to the dithering vibration frequency due to the use of a bulky system. This can be greatly improved by designing a very light weight laser head with an embedded PZT. Modulating the laser driving current might also be another promising way to achieve higher bandwidth.

\section{ACKNOWLEDGMENT}

The authors would like to thank Agence Nationale de la Recherche (ANR) : LabCom CapIRO in cooperation with ACOEM, Thierry MAZOYER

\section{REFERENCES}

[1] S. Donati, "Developing self-mixing interferometry for instrumentation and measurements," Laser \& Photonics Reviews, vol. 6, no. 3, pp. 393417, 2012.

[2] T. Taimre, M. Nikolić, K. Bertling, Y. L. Lim, T. Bosch, and A. D. Rakić, "Laser feedback interferometry: a tutorial on the self-mixing effect for coherent sensing," Adv. Opt. Photon., vol. 7, no. 3, pp. 570-631, Sep 2015.

[3] F. P. Mezzapesa, A. Ancona, T. Sibillano, F. D. Lucia, M. Dabbicco, P. M. Lugarà, and G. Scamarcio, "High-resolution monitoring of the hole depth during ultrafast laser ablation drilling by diode laser self-mixing interferometry," Opt. Lett., vol. 36, no. 6, pp. 822-824, Mar 2011.

[4] G. Giuliani, S. Bozzi-Pietra, and S. Donati, "Self-mixing laser diode vibrometer," Measurement Science and Technology, vol. 14, no. 1, pp. 24-32, nov 2002.

[5] Z. Wei, W. Huang, J. Zhang, X. Wang, H. Zhu, T. An, and X. Yu, "Obtaining scalable fringe precision in self-mixing interference using an even-power fast algorithm," IEEE Photonics Journal, vol. 9, no. 4, pp. 1-11, Aug 2017.

[6] C. Jiang, C. Li, S. Yin, and Z. Huang, "Multiple self-mixing interferometry algorithm based on phase modulation for vibration measurement," Optical and Quantum Electronics, vol. 49, no. 3, p. 111, Feb 2017.

[7] C. Bes, G. Plantier, and T. Bosch, "Displacement measurements using a self-mixing laser diode under moderate feedback," IEEE Transactions on Instrumentation and Measurement, vol. 55, no. 4, pp. 1101-1105, Aug 2006.

[8] O. D. Bernal, U. Zabit, and T. Bosch, "Study of laser feedback phase under self-mixing leading to improved phase unwrapping for vibration sensing," IEEE Sensors Journal, vol. 13, no. 12, pp. 4962-4971, Dec 2013.

[9] Y. Fan, Y. Yu, J. Xi, and J. F. Chicharo, "Improving the measurement performance for a self-mixing interferometry-based displacement sensing system," Appl. Opt., vol. 50, no. 26, pp. 5064-5072, Sep 2011.

[10] A. L. Arriaga, F. Bony, and T. Bosch, "Real-time algorithm for versatile displacement sensors based on self-mixing interferometry," IEEE Sensors Journal, vol. 16, no. 1, pp. 195-202, Jan 2016.

[11] S. Merlo and S. Donati, "Reconstruction of displacement waveforms with a single-channel laser-diode feedback interferometer," IEEE Journal of Quantum Electronics, vol. 33, no. 4, pp. 527-531, April 1997.

[12] A. Ehtesham, U. Zabit, O. D. Bernal, G. Raja, and T. Bosch, "Analysis and implementation of a direct phase unwrapping method for displacement measurement using self-mixing interferometry," IEEE Sensors Journal, vol. 17, no. 22, pp. 7425-7432, Nov 2017. 
[13] U. Zabit, O. D. Bernal, S. Amin, M. F. Qureshi, A. H. Khawaja, and T. Bosch, "Spectral processing of self-mixing interferometric signal phase for improved vibration sensing under weak-and moderatefeedback regime," IEEE Sensors Journal, vol. 19, no. 23, pp. 11151 11 158, December 2019.

[14] D. Melchionni, A. Magnani, A. Pesatori, and M. Norgia, "Development of a design tool for closed-loop digital vibrometer," Appl. Opt., vol. 54 no. 32, pp. 9637-9643, Nov 2015.

[15] A. Magnani, D. Melchionni, A. Pesatori, and M. Norgia, "Selfmixing digital closed-loop vibrometer for high accuracy vibration measurements," Optics Communications, vol. 365, pp. 133 - 139, 2016. [Online]. Available: http://www.sciencedirect.com/science/article/ pii/S0030401815303394

[16] S. Donati and M. Norgia, "Self-mixing interferometer with a laser diode: Unveiling the fm channel and its advantages respect to the am channel," IEEE Journal of Quantum Electronics, vol. 53, no. 5, pp. 1-10, Oct 2017.

[17] V. Contreras, J. Lonnqvist, and J. Toivonen, "Edge filter enhanced self-mixing interferometry," Opt. Lett., vol. 40, no. 12, pp. 2814 2817, Jun 2015. [Online]. Available: http://ol.osa.org/abstract.cfm?URI= ol-40-12-2814

[18] M. Norgia, D. Melchionni, and S. Donati, "Exploiting the fm-signal in a laser-diode smi by means of a mach-zehnder filter," IEEE Photonics Technology Letters, vol. 29, no. 18, pp. 1552-1555, Sep. 2017.

[19] F. J. Azcona, R. Atashkhooei, S. Royo, J. M. Astudillo, and A. Jha, "A nanometric displacement measurement system using differential optical feedback interferometry," IEEE Photonics Technology Letters, vol. 25 no. 21, pp. 2074-2077, Nov 2013.

[20] M. C. Giordano, S. Mastel, C. Liewald, L. L. Columbo, M. Brambilla, L. Viti, A. Politano, K. Zhang, L. Li, A. G. Davies, E. H. Linfield, R. Hillenbrand, F. Keilmann, G. Scamarcio, and M. S. Vitiello, "Phaseresolved terahertz self-detection near-field microscopy," Opt. Express, vol. 26, no. 14, pp. 18423-18435, Jul 2018. [Online]. Available: http://www.opticsexpress.org/abstract.cfm?URI=oe-26-14-18423

[21] W. Xia, Q. Liu, H. Hao, D. Guo, M. Wang, and X. Chen, "Sinusoidal phase-modulating self-mixing interferometer with nanometer resolution and improved measurement velocity range," Appl. Opt., vol. 54, no. 26, pp. 7820-7827, Sep 2015. [Online]. Available: http: //ao.osa.org/abstract.cfm?URI=ao-54-26-7820

[22] O. D. Bernal, U. Zabit, and T. Bosch, "Classification of laser self-mixing interferometric signal under moderate feedback," Appl. Opt., vol. 53, no. 4, pp. 702-708, Feb 2014.

[23] A. Magnani, A. Pesatori, and M. Norgia, "Self-mixing vibrometer with real-time digital signal elaboration," Appl. Opt., vol. 51, no. 21, pp. 5318-5325, Jul 2012. [Online]. Available: http://ao.osa.org/abstract. cfm?URI=ao-51-21-5318

[24] N. Sayiner, H. V. Sorensen, and T. R. Viswanathan, "A level-crossing sampling scheme for a/d conversion," IEEE Transactions on Circuits and Systems II: Analog and Digital Signal Processing, vol. 43, no. 4, pp. 335-339, April 1996.

[25] C. Vezyrtzis and Y. Tsividis, "Processing of signals using level-crossing sampling," in 2009 IEEE International Symposium on Circuits and Systems, May 2009, pp. 2293-2296.

[26] F. Marvasti, Nonuniform sampling: theory and practice. Springer US 2001.

[27] P. W. Jungwirth and A. D. Poularikas, "Improved sayiner level crossing adc," in Thirty-Sixth Southeastern Symposium on System Theory, 2004. Proceedings of the, March 2004, pp. 379-383.

[28] T. Wang, D. Wang, P. J. Hurst, B. C. Levy, and S. H. Lewis, "A level-crossing analog-to-digital converter with triangular dither," IEEE Transactions on Circuits and Systems I: Regular Papers, vol. 56, no. 9, pp. 2089-2099, Sep. 2009.

[29] G. Plantier, C. Bes, and T. Bosch, "Behavioral model of a self-mixing laser diode sensor,' IEEE Journal of Quantum Electronics, vol. 41, no. 9 pp. 1157-1167, Sep. 2005.

[30] U. Zabit, O. D. Bernal, and T. Bosch, "Self-mixing laser sensor for large displacements: Signal recovery in the presence of speckle," IEEE Sensors Journal, vol. 13, no. 2, pp. 824-831, Feb 2013.

[31] G. Giuliani and M. Norgia, "Laser diode linewidth measurement by means of self-mixing interferometry," IEEE Photonics Technology Letters, vol. 12, no. 8, pp. 1028-1030, Aug 2000.

[32] O. D. Bernal, U. Zabit, and T. M. Bosch, "Robust method of stabilization of optical feedback regime by using adaptive optics for a self-mixing micro-interferometer laser displacement sensor," IEEE Journal of Selected Topics in Quantum Electronics, vol. 21, no. 4, pp. 336-343, July 2015.
[33] A. A. Siddiqui, U. Zabit, O. D. Bernal, G. Raja, and T. Bosch, "All analog processing of speckle affected self-mixing interferometric signals," IEEE Sensors Journal, vol. 17, no. 18, pp. 5892-5899, Sep. 2017. 